\title{
Self-Reinforced Hybrid Polyethylene/MCM-41 Nanocomposites: In-Situ Polymerisation and Effect of MCM-41 Content on Rigidity
}

\author{
João M. Campos ${ }^{1}$, João Paulo Lourenço², Ernesto Pérez³ \\ María L. Cerrada ${ }^{3, *}$, and M. Rosário Ribeiro ${ }^{1, *}$ \\ ${ }^{1}$ Instituto de Ciência e Engenharia de Materiais e Superfícies \& Departamento de Engenharia Química e Biológica, \\ Instituto Superior Técnico - Universidade Técnica de Lisboa, Av. Rovisco Pais 1, 1049-001 Lisboa, Portugal \\ ${ }^{2}$ Centro de Investigação em Química do Algarve, Departamento de Química, \\ Bioquímica e Farmácia Faculdade de Ciências e Tecnologia - Universidade do Algarve, \\ Campus de Gambelas, 8005-139 Faro, Portugal \\ ${ }^{3}$ Instituto de Ciencia y Tecnología de Polímeros (CSIC), Juan de la Cierva 3, 28006 Madrid, Spain
}

\begin{abstract}
The synthesis of self-reinforced polyethylene-based materials prepared by in-situ polymerisation is described. The methodology developed uses MCM-41 mesoporous material in a triple role: as catalyst carrier for ethylene polymerisation within its pores and channels in a first stage, as nanofiller of the formed polyethylene matrix during its useful lifetime and, finally, as promoter for long-term waste disposal. As evidenced by FTIR analysis, when the polymer is formed under these confined conditions different interactions between the MCM- 41 material and the polyethylene matrix occur, when compared to simple blends. The influence of the filler content on the rigidity of the resulting nanocomposites is analysed by microhardness measurements and corroborated by the storage modulus values: a significant increase in rigidity is observed as the filler contents rises. In addition, thermogravimetric studies show interesting features concerning the degradability of these materials. The catalytic action of MCM-41 during degradation involves a reduction of the energetic requirements for their cracking and, therefore, a positive environmental impact.
\end{abstract}

Keywords: MCM-41, Metallocene Catalysts, Microhardness, Nanocomposites, Polyethylene.

\section{INTRODUCTION}

Organic-inorganic hybrid composite materials present extraordinary properties arising from the synergism between both components. The composites obtained from the incorporation of inorganic constituent in organic polymers are probably the most interesting ones, showing remarkable changes in their thermal, ${ }^{1}$ mechanical, ${ }^{2-5}$ electrical $^{6}$ and magnetic ${ }^{7}$ properties compared with those found in the neat polymers. The ones scientifically well explored and reported are those when inorganic filler size is within the microscale. ${ }^{8-12}$ The decrease in size of the inorganic component down to the nano-dimension and the increase in the interfacial area result in a new kind of materials that exhibit improved properties: the nanocomposites. Their preparation and the study of their behaviour have imposed a challenge in the last decade. ${ }^{13}$ Zeolites

*Authors to whom correspondence should be addressed. and mesoporous materials are particularly interesting to be used as the inorganic phase for the preparation of this type of nanocomposites. These present stable 3D framework structures that can resist to the forces produced by the intercalated polymers, which tend to separate the two constituents, one of the key problems to overcome in the preparation of these nanocomposites.

Two primary methods exist for the preparation of nanocomposites from porous inorganic materials: ${ }^{13}$ (i) direct threading of preformed polymer through the inorganic host channels (soluble and melting polymers), usually limited by the size, conformation, and diffusion behaviour of the polymers, and (ii) the in-situ polymerisation in the pores and channels of the inorganic hosts. In-situ polymerisation gives better filler dispersion than simple melt preparation, especially at high filler contents, and provides a promising strategy for obtaining nanocomposites with desirable properties. ${ }^{14}$ The research papers published recently reported various methods for the 
synthesis of nanocomposites combining natural and synthetic zeolites, or other layered materials, with in-situ polymerisation of olefins. ${ }^{14-19}$

The M41S materials and, in particular, the MCM-41 one with a hexagonal array of unidirectional pores show promising results when used as carriers for single-site olefin polymerisation catalysts. Moreover, they can keep steadily their 3D frameworks and the polymers formed under the extreme confinement imposed by these carriers might show unusual morphologies and mechanical properties. $^{20}$

The majority of the articles published until now has been focused mainly on: (i) the catalytic aspects, that is, the effects on polymerisation activities of the carrier composition and of its interactions with the catalyst and cocatalyst, or (ii) the unusual morphological properties of the polyolefins formed under the extreme confinement imposed by the catalyst support. Therefore, several reports dealing with polymerisation of olefins using transition metal catalysts immobilised in MCM-41 supports have already been described in the literature. ${ }^{21-27}$ However, research is not primarily focused on the potential of these materials (when associated with in-situ polymerisation technique) for the preparation of nanocomposites and there are scarce articles in literature associated with evaluation of bulk properties of the resulting materials. ${ }^{28-30}$ Thus, the aim of this work is to use MCM- 41 mesoporous materials for the preparation of polyolefinic nanocomposites, with some of their features being preliminarily analysed. Here, MCM-41 material plays a triple role: in a first stage, as a catalyst carrier making possible the ethylene polymerisation within its pores and channels; in a second one as nanofiller of the polyethylene matrix formed; and, at last, as promoter for the degradation of the material once its service lifetime is over. Accordingly, to cover these three different aspects this article describes the preparation method and the results found from the study of the effect of MCM-41 content, ranged from 2 to $28 \mathrm{wt} \%$, on the mechanical response of the nanocomposites (by microhardness $(\mathrm{MH})$ and storage modulus determination), as well as those related to their thermal stability. The $\mathrm{MH}$ estimation allows a fast, reliable and bulk-comparable analysis of how the incorporation of mesoporous MCM-41 influences on rigidity of the resulting nanocomposites. It is noticeable that typical contents of nanofillers added into polymeric matrices are around $10 \mathrm{wt} \%$ or lower, because of agglomeration problems and to maintain manufacturing costs as low as possible. However, this work also deals with higher incorporations, analysing the resultant homogeneity and the influence of high contents on the final mechanical properties. In this case, costs are not an issue because the mesoporous component is an element in the synthetic protocol used because of its key role as catalyst carrier.

\section{EXPERIMENTAL DETAILS}

\subsection{Reagents}

$\mathrm{Cp}_{2} \mathrm{ZrCl}_{2} \quad$ (Aldrich) and methylaluminoxane (MAO, $10 \% \mathrm{~m} / \mathrm{v}$ toluene solution, Aldrich) were used as received. Commercial toluene (Petrogal) was purified by refluxing over sodium and benzophenone, before distillation. Nitrogen and ethylene (Air Liquide) were dried using absorption columns containing molecular sieves $13 \times$ and $4 \mathrm{~A}$.

\subsection{Preparation of the Hybrid PE/MCM-41 Nanocomposite Materials}

\subsubsection{Synthesis of the MCM-41 Mesoporous Supports}

Purely siliceous and aluminated MCM-41 mesoporous materials have been used as supports for the preparation of heterogeneous polymerisation catalysts. The synthesis procedure and the characterisation data of siliceous MCM-41 and aluminium-containing MCM-41 (direct synthesis) are described extensively elsewhere, ${ }^{31}$ their structural parameters being reported in Table I. The three aluminated MCM-41 supports have Si/Al ratios of 47,30 and 16, as determined by bulk elemental analysis. These will be referred as M47, M30 and M16, respectively. Purely siliceous MCM-41 is labelled as MSI.

\subsubsection{Preparation of Polymerisation Catalyst Supported on the Mesoporous Materials}

Olefin polymerisation catalysts were prepared by combining $\mathrm{Cp}_{2} \mathrm{ZrCl}_{2}$ with the mesoporous supports MSI, M47, M30 and M16, using a simplified version of the wellknown impregnation method. Typically, $0.5 \mathrm{~g}$ of the mesoporous solid is contacted with a solution of the metallocene $1.7 \times 10^{-3} \mathrm{M}$ in toluene, for $16 \mathrm{~h}$, in a Schlenk flask with magnetic stirring and concealed from ambient light. A description and discussion of this experimental procedure can be found in a previous paper. ${ }^{31}$ Catalysts derived from supports MSI, M47, M30 and M16, are referred, respectively, as ZIR-MSI, ZIR-M47, ZIR-M30 and ZIR-M16.

\subsubsection{Polymerisation}

The polymerisation reactor consists in a $250 \mathrm{~cm}^{3}$ bottle for pressure reactions (Wilmad LabGlass LG-3921), and a

Table I. Structural parameters of the four mesoporous solids: $S_{\mathrm{BET}}$ : specific surface area; $V_{\mathrm{p}}$ : specific pore volume and $D_{\mathrm{p}}$ : average pore diameter $\left(D_{\mathrm{p}}=4 \mathrm{~V}_{\mathrm{p}} / S_{\mathrm{BET}}\right)$.

\begin{tabular}{lcccc}
\hline Support & Si/Al & $S_{\text {BET }}\left(\mathrm{m}^{2} / \mathrm{g}\right)$ & $V_{\mathrm{p}}\left(\mathrm{cm}^{3} / \mathrm{g}\right)$ & $D_{\mathrm{p}}(\AA)$ \\
\hline MSI & - & 1007 & 0.85 & 33.8 \\
M47 & 47 & 975 & 0.80 & 32.8 \\
M30 & 30 & 1057 & 0.87 & 32.9 \\
M16 & 16 & 1092 & 0.82 & 30.0 \\
\hline
\end{tabular}


magnetic stirrer. This reactor is purged with vacuum/ $\mathrm{N}_{2}$, loaded with toluene and placed in a water bath with controlled temperature. Nitrogen is replaced by ethylene and the appropriate amount of MAO cocatalyst solution is injected into the reactor. The suspension of mesoporous solid containing the catalyst is vigorously stirred and the equivalent to $2 \times 10^{-6} \mathrm{~mol} \mathrm{Zr}$ is injected into the reactor by measuring an appropriate volume of this fine and homogeneous suspension. After 30 minutes of polymerisation, the reactor is discharged and the polymer is precipitated over methanol acidified with $\mathrm{HCl}$, and further washed twice with fresh methanol before drying. The different hybrid inorganic/organic nanocomposite materials thus obtained are labelled as HDPE/MCM-41(x), the $x$ being the weight content in MCM-41.

In addition, a HDPE homopolymer is synthesised under homogeneous conditions, similar to those previously described, ${ }^{31}$ to fully evaluate the effect of the mesoporous MCM-41 in the inherent characteristics of these materials. In another experiment, a reactor mixture of HDPE and MCM-41 is prepared by adding $50 \mathrm{mg}$ of dry MCM-41 powder (dispersed in toluene) to the reactor prior to ethylene polymerisation in homogeneous media (using soluble $\mathrm{Cp}_{2} \mathrm{ZrCl}_{2} / \mathrm{MAO}$ catalytic system).

\subsection{FTIR Spectroscopy}

A Thermo-Nicolet Nexus FTIR equipment, in transmission mode, was used to establish a calibration pattern and, consequently, determine accurately the composition from the different HDPE/MCM-41( $x$ ) nanocomposites synthesised in-situ. Several HDPE/MCM-41 blends with different mesoporous MCM-41 contents, up to $\sim 15 \%(\mathrm{w} / \mathrm{w})$, were prepared for this purpose. Thus, powdered homogeneous HDPE was randomly mixed with the mesoporous solid, and thin films were prepared in a SpecAc press with heating plates. Stainless steel moulds with spacers were used to obtain films with uniform thickness from HDPE/MCM-41 mixtures under pressure at $150{ }^{\circ} \mathrm{C}$. In all of the films, spectra were taken at different spots (at least four different measurements) to ensure the homogeneity in the MCM-41 distribution within the polymeric matrix. The spectra collected for each film were normalised in relation to the intensity of the absorption band centred at $720 \mathrm{~cm}^{-1}$, which is associated with main polyolefin backbone, and the final average spectrum is computed.

Samples of the synthesised hybrid materials were also obtained as films for the FTIR evaluation, as described above. The weight composition in nanofiller is estimated for the different in-situ polymerised nanocomposites through the calibration attained from the HDPE/MCM-41 blends and their two characteristic absorption bands.

A Pike Technology Smart Miracle ATR accessory was coupled to the FTIR equipment, in order to analyse the non-soluble fractions resulting from the solvent extraction experiments.

\subsection{Preparation of Specimens}

The hybrid HDPE/MCM-41 $(x)$ nanocomposites were obtained as thick films (around $350 \mu \mathrm{m}$ ) for the thermal, dynamic mechanical, microhardness and thermogravimetric measurements, by compression moulding in a Collin press between hot plates at $170{ }^{\circ} \mathrm{C}$, at a pressure of 1.5 MPa for $5 \mathrm{~min}$. Each of the HDPE/MCM-41( $x)$ specimens was crystallised after its melting in the press by a fast quench in cold water.

\subsection{X-ray Measurements}

Room-temperature X-ray diffraction experiments were performed by employing synchrotron radiation $(\lambda=$ $0.150 \mathrm{~nm}$ ) in the soft-condensed matter beamline A2 at HASYLAB (Hamburg, Germany). A CCD detector was used covering the approximate $2 \theta$ range from 1 to $30^{\circ}$.

\subsection{TEM Analysis}

TEM images were obtained in Hitachi H8100 equipment. Nanocomposite samples were deposited in a $\mathrm{Cu} /$ polymer grid sample holder.

\subsection{Solvent Extraction}

A portion of $\sim 300 \mathrm{mg}$ of the hybrid material is placed in a filtering thimble and extracted with a boiling mixture of xylene isomers $\left(\sim 140{ }^{\circ} \mathrm{C}\right)$ for 10 hours, under $\mathrm{N}_{2}$ atmosphere. The soluble polymer fraction is precipitated and washed with methanol and dried. The insoluble inorganic fraction is washed with methanol and dried.

\subsection{Thermal Characterisation}

The effect of MCM-41 content on melting temperature $\left(T_{\mathrm{m}}\right)$ and crystallinity $\left(f_{\mathrm{c}}\right)$ is preliminary estimated by calorimetric analyses. These were carried out in a PerkinElmer DSC7 calorimeter, connected to a cooling system and calibrated with different standards. The sample weights ranged from 6 to $7.5 \mathrm{mg}$. A temperature range from $-50{ }^{\circ} \mathrm{C}$ to $150{ }^{\circ} \mathrm{C}$ has been studied and the used heating rate was $10{ }^{\circ} \mathrm{C} \mathrm{m^{-1 }}$. For crystallinity determinations, enthalpy is normalized taking into account the real amount of polyethylene within the nanocomposite and a value of $290 \mathrm{~J} \mathrm{~g}^{-1}$ is taken as the enthalpy of fusion of a perfectly crystalline material. ${ }^{32}$

\subsection{Microhardness Measurements}

A Vickers indentor attached to a Leitz microhardness tester was used to perform microindentation measurements undertaken at $23{ }^{\circ} \mathrm{C}$. A contact load of $0.98 \mathrm{~N}$ and a contact time of $25 \mathrm{~s}$ were employed. Microhardness, 
$\mathrm{MH}$, values (in $\mathrm{MPa}$ ) were calculated according to the relationship: ${ }^{33}$

$$
\mathrm{MH}=2 \sin 68^{\circ} P / d^{2}
$$

where $P$ (in $\mathrm{N}$ ) is the contact load and $d$ (in $\mathrm{mm}$ ) is the diagonal length of the projected indentation area. Diagonals were measured in the reflected light mode within $30 \mathrm{~s}$ of load removal, using a digital eyepiece equipped with a Leitz computer-counter-printer (RZA-DO).

\subsection{Elastic Modulus Estimation}

The storage modulus $\left(\mathrm{E}^{\prime}\right)$ associated with the elastic contribution to the bulk complex modulus for each sample was determined in a tensile mode at $3 \mathrm{~Hz}$ using a Polymer Laboratories MK II dynamic mechanical thermal analyser, DMTA, to compare the response in the bulk with respect to that superficial one given by microhardness for the different nanocomposites.

\subsection{Thermogravimetric Analysis}

The weight loss was estimated by thermogravimetry using a TA Instruments TGA Q500 equipment working under an inert atmosphere. The equipment was calibrated according to standard protocols. The sample weights ranged from 4 to $6 \mathrm{mg}$, and the heating rate was $10{ }^{\circ} \mathrm{C} \mathrm{min}^{-1}$.

\section{RESULTS AND DISCUSSION}

The preparation of hybrid organic-inorganic nanocomposites by in-situ polymerisation may involve both the dispersion of the nanoscaled filler in the reaction medium before and during polymerisation ${ }^{34}$ or the immobilisation of the polymerisation catalyst onto the nanofiller surface. ${ }^{35}$ The route here followed takes advantage of the unique properties of MCM-41 materials and involves immobilisation of catalyst on this mesoporous material, allowing therefore polymerisation from the filler surface.

The zirconocene catalyst was immobilised on the MCM-41 material via a direct impregnation route. Under the tested polymerisation conditions no extraction occurs.
Polymerisation activity can significantly change by varying from purely siliceous to an aluminated support, as shown in a previous research. ${ }^{31}$ Accordingly, different MCM-41based supports and polymerisation conditions have been carefully selected in this work to control the activity level and to, consequently, enable the preparation of hybrid nanocomposite materials with different contents in inorganic nanofillers.

Several ethylene polymerisations have been performed with catalysts derived from both pure siliceous (ZIRMSI) and aluminated supports (ZIR-M47, ZIR-M30 and ZIR-M16). Table II shows the experimental conditions that allow the successful HDPE/MCM-41 $(x)$ nanocomposites preparation as well as yields and the estimated filler contents.

\subsection{Structural Characterisation}

Figure 1 shows the X-ray diffraction profiles in the distinctive region of MCM-41 materials for the different specimens here analysed in order to establish if some polyethylene chains have been synthesised within its pores and channels. The initial MCM-41 exhibits a diffraction pattern characteristic of mesoporous hexagonally arranged structures (reflections $\{100\},\{110\},\{200\}$ and $\{210\}$ ). The different nanocomposites present the same diffractions indicating that the long-range structure is retained after polymerisation. However, differences in intensity and in width are found for the various samples. The former feature occurs because MCM-41 derivatives are not the major component in the material whereas the second one is ascribed to the fact that polyethylene has been inserted within the pores and channels, slightly deforming their original well-ordered long-range structure. The width of the two-dimensional MCM-41 hexagonal structure would be unchanged if polymeric macrochains were out, therefore this width variation confirms the insertion of polyethylene during the in situ polymerisation.

Figure 2(a) shows the FTIR spectra for the purely siliceous MCM-41 and compares it with the spectra obtained for a set at similar filler contents of reference HDPE/MCM-41 blends and in-situ synthesised

Table II. Experimental conditions, reaction yields and MCM-41 contents (estimated by FTIR and thermogravimetric analysis) for the preparation of the self-reinforced HDPE/MCM-41 $(x)$ nanocomposites.

\begin{tabular}{|c|c|c|c|c|c|c|c|c|}
\hline \multirow[b]{2}{*}{ Nanocomposite } & \multirow[b]{2}{*}{ Catalyst } & \multirow{2}{*}{$\begin{array}{c}\mathrm{Cp}_{2} \mathrm{ZrCl}_{2} \text { load in } \\
\text { support }\left(10^{-6} \mathrm{~mol} / \mathrm{g}\right)\end{array}$} & \multirow[b]{2}{*}{ Amount of support (mg) } & \multirow[b]{2}{*}{$\mathrm{Al} / \mathrm{Zr}$} & \multirow[b]{2}{*}{ Yield (mg) } & \multicolumn{3}{|c|}{ MCM-41 (\% wt) } \\
\hline & & & & & & Support (mg)/yield (mg) & FTIR & TGA \\
\hline HDPE & $\mathrm{Cp}_{2} \mathrm{ZrCl}_{2}{ }^{a}$ & - & - & 500 & 2200 & - & - & 0 \\
\hline HDPE/MCM-41(3) & ZIR-M30 & 50 & 40 & 1500 & 1560 & 3 & 3 & 3 \\
\hline HDPE/MCM-41(4) & ZIR-M16 & 50 & 41 & 500 & 950 & 4 & 4 & 4 \\
\hline HDPE/MCM-41(6) & ZIR-M47 & 50 & 41 & 500 & 680 & 6 & 6 & 5 \\
\hline HDPE/MCM-41(14) & ZIR-MSI & 13 & 160 & 1500 & 1160 & 14 & 13 & 16 \\
\hline HDPE/MCM-41(23) & ZIR-MSI & 13 & 166 & 500 & 720 & 23 & - & 24 \\
\hline HDPE/MCM-41(28) & ZIR-MSI & 13 & 157 & 500 & 560 & 28 & - & 33 \\
\hline
\end{tabular}

${ }^{a} 1 \times 10^{-6} \mathrm{~mol}$. 

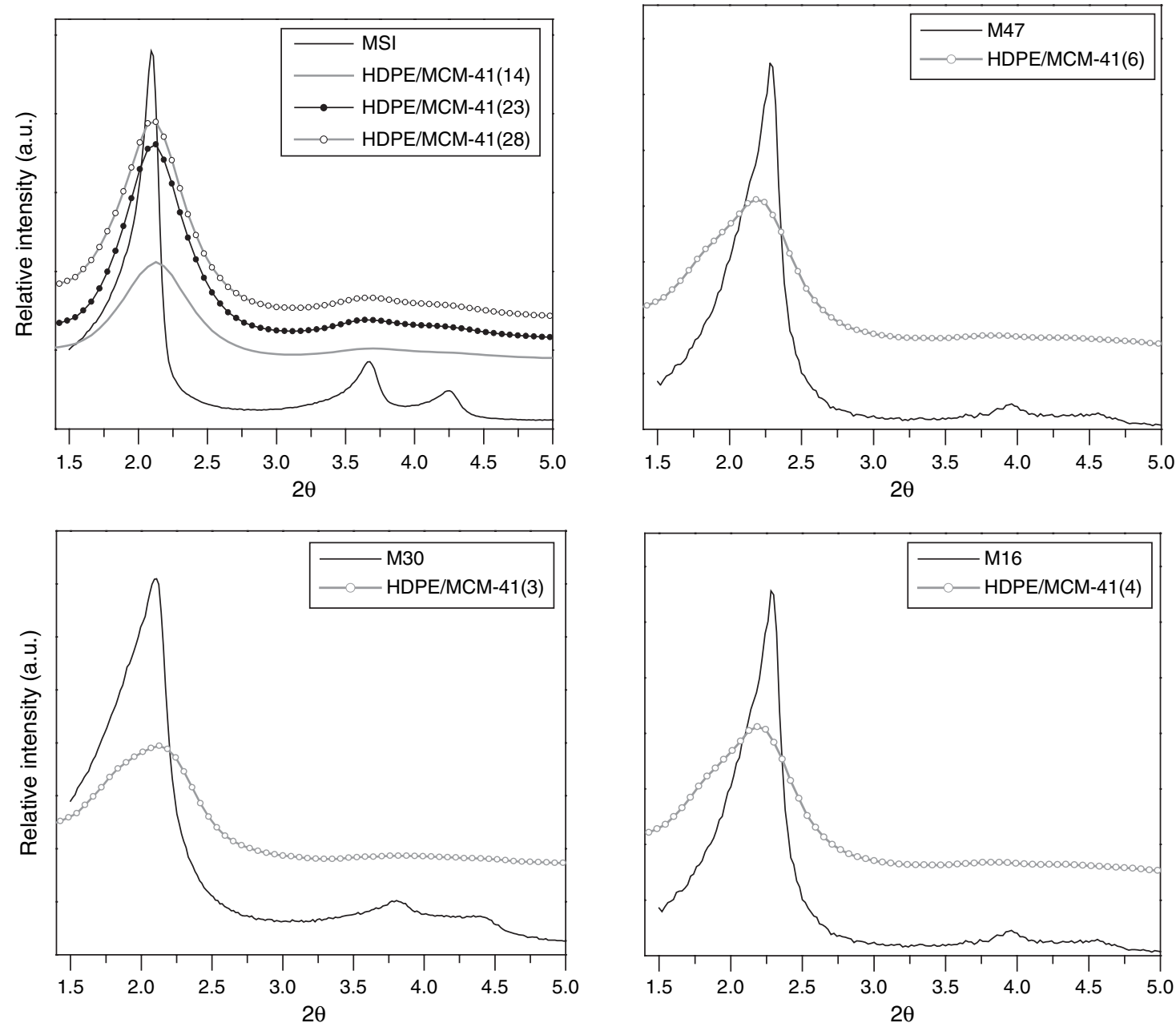

Fig. 1. $\mathrm{X}$ ray profiles in the region of middle angles for the distinct supports and HDPE/MCM-41(x) nanocomposites.

HDPE/MCM-41(x) nanocomposites. Pure HDPE does not present any distinctive band in the MCM-41 fingerprint FTIR region. However, the HDPE/MCM-41(x) hybrids display two strong absorption bands. These are centred at $\sim 1245$ (L1) and $\sim 1075 \mathrm{~cm}^{-1}$ (L2) and are due, respectively, to the outer and inner asymmetrical stretch vibrations of the $\mathrm{TO}_{4}$ tetrahedra $(\mathrm{T}=\mathrm{Si}$ or $\mathrm{Al})$ present in the siliceous material. ${ }^{36}$ Figure 2(b) presents in detail the region of absorption band L1. A shift to lower wavenumbers is evident for the absorptions in the hybrid nanocomposites compared to those found in the blends, at a given inorganic content. This feature indicates
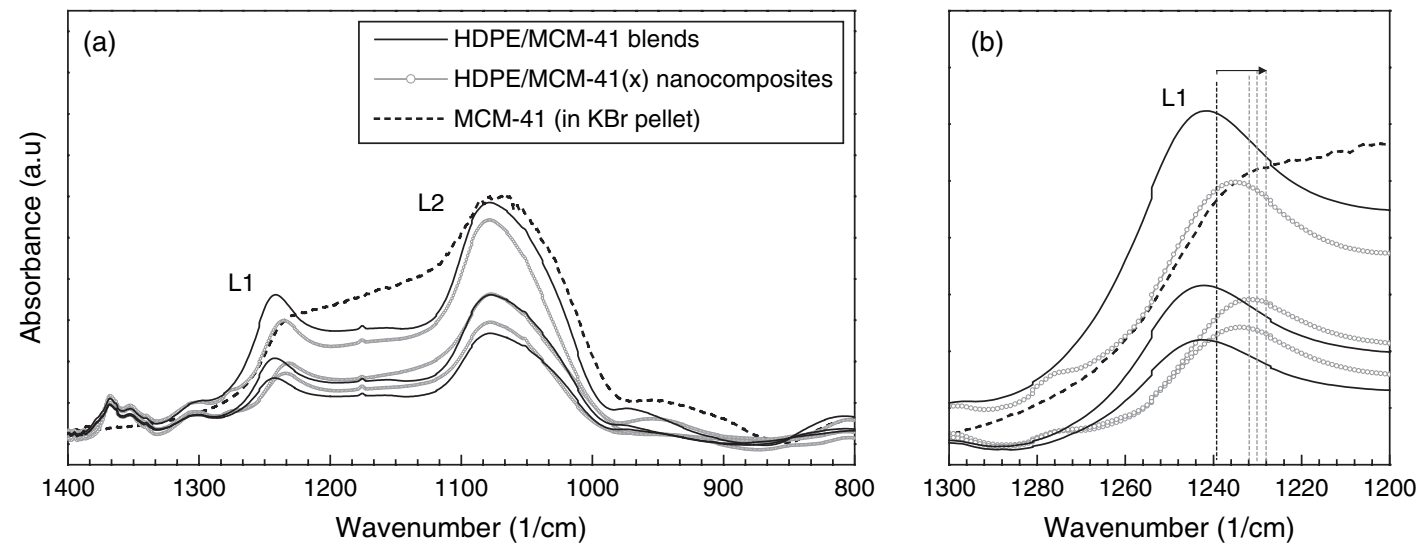

Fig. 2. (a) FTIR spectra for the filler MCM-41, for several HDPE/MCM-41 blends and corresponding HDPE/MCM-41( $x$ ) nanocomposites polymerised in-situ at similar filler content; (b) Detail of the shift that occurs in band L1. 
some changes in the phase interactions existing in a polyethylene/MCM-41 hybrid material when it is prepared by in-situ polymerisation or by simple blending. These differences could be ascribed to the fact that in the in-situ prepared nanocomposites there are polyethylene chains within the MCM-41 channels favouring strong interactions between the two components and, consequently, weakening the specific vibrations of the $\mathrm{TO}_{4}$ tetrahedra. On the other hand, no changes in the characteristic polyethylene vibrations are observed in the blends when macromolecules are just surrounding the mesoporous material.

The intensity increases in bands L1 and L2 as the inorganic filler content does. Spectra taken in different spots of films of the HDPE/MCM-41 blends show that, up to a concentration of $\sim 12 \%$ in nanofiller, these are homogeneous. A linear relation is observed in this region between the intensity of band L1 (or L2) and the contents in nanofiller (Fig. 3). However, for higher concentrations, the nanofillers tend to aggregate and certain macroscopic blend heterogeneity is observed.

The nanofiller distribution homogeneity within the nanocomposites was also checked. Similar L1 and L2 intensities are observed at the different sections tested. However, films tend to become opaque and a saturation effect is observed at nanofiller contents around $12 \mathrm{wt} \%$. To estimate the composition of the nanocomposites above this concentration range, a controlled dilution procedure by blending with HDPE homopolymer has been used. This method proved to be accurate for inorganic filler concentrations up to $14 \%$. Results on Table II show good agreement between the compositions of the HDPE/MCM-41(x) nanocomposites estimated by FTIR analysis and those obtained considering the amount of mesoporous solid loaded into the reactor and the mass of product formed, in this concentration range.

TEM micrographs provide information about either particle distribution or size of agglomerates of MCM-41

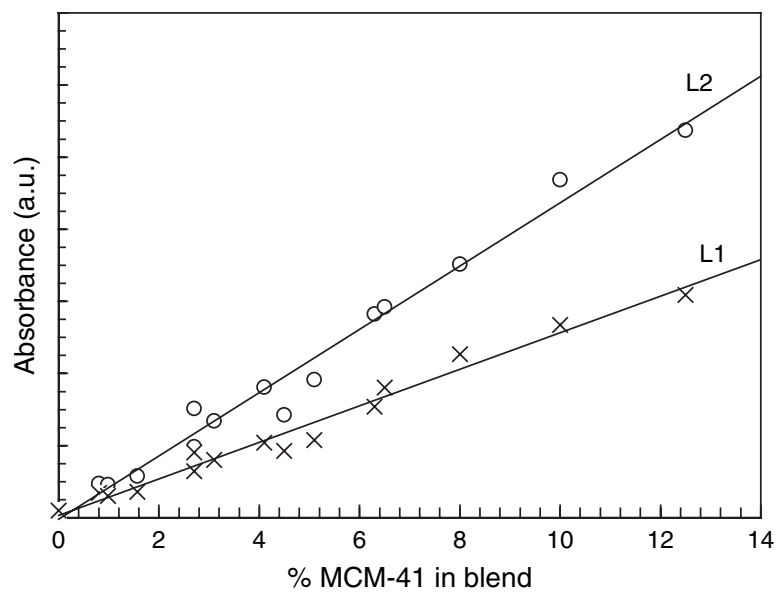

Fig. 3. Relationship between the intensity exhibited by the absorption bands centred at $\sim 1245^{-1}$ (L1) and $\sim 1075 \mathrm{~cm}^{-1}$ (L2) and the MCM-41 concentration in HDPE/MCM-41 blends. within the polyethylene matrix. Moreover, these micrographs and some additional experiments have been also performed to confirm the presence of the channel structure in the nanocomposites. The picture at top of Figure 4 shows that particles are uniformly dispersed within the polyethylene matrix, some aggregates being also observed. These features were observed in the nanometric scale, for the HDPE/MCM-41(14) nanocomposite. On the other hand, the picture in the middle of Figure 4 clearly

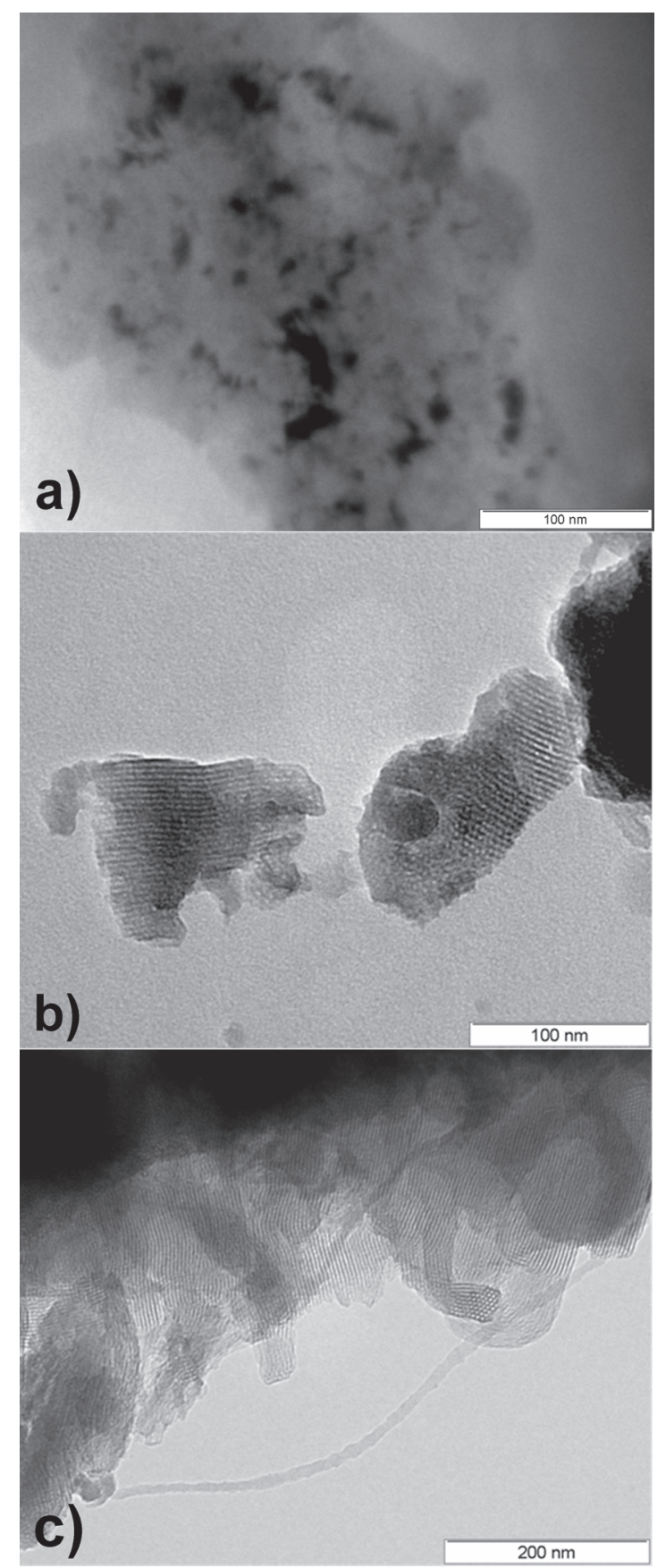

Fig. 4. TEM micrographs: (a) HDPE/MCM-41(14); (b) MCM-41; and (c) non-soluble fraction obtained by extraction by xylene from HDPE/MCM-41(23). 
shows the ordered channel structure existing in the initial MCM-41. We believe that these nanocomposites consist in polyethylene chains incorporated inside channels of the MCM-41 derivatives (as supported by the X-ray results discussed above) in addition to some others chains surrounding the nanofillers. To provide more evidences to this assumption, the nanocomposites have been subjected to an extraction process using xylene as solvent at $140{ }^{\circ} \mathrm{C}$ for 10 hours to separate the soluble outer polyethylene chains from the non-soluble residue composed of inorganic MCM-41 nanofillers and the inner polyethylene chains located within its channels. Picture at bottom of Figure 4 evidently shows the ordered channel structure remaining in the non-soluble fraction and the presence of small fibres with thickness of some tens of nanometers (still remaining after extraction with xylene), supporting the occurrence of intrapore polymerisation.

The initial nanocomposites and the products obtained from the soluble and non-soluble fractions were also surveyed by FTIR spectroscopy in ATR mode. Figure 5 represents the results found considering the nanocomposite HDPE/MCM-41(23). Its spectrum shows the characteristics bands of polyethylenes (around 2900, 1400-1500 and $\left.700-800 \mathrm{~cm}^{-1}\right)$ and those related to the MCM-41 fingerprint (at $\sim 1245$ and $\sim 1075 \mathrm{~cm}^{-1}$ ). The soluble fraction basically only exhibits the bands related to pure polyethylene. However the non-soluble part shows both the bands belonging to MCM-41 and those to PE, attributed to remaining polyethylene chains within channels. Moreover when the same extraction process was carried out over a composite obtained by using the MCM- 41 only as filler in the polymerisation reactor (and not as catalyst carrier) no traces of PE were found in the non-soluble fraction. In this case, no PE chains are expected to form inside the MCM-41 channels, and accordingly the removal of the

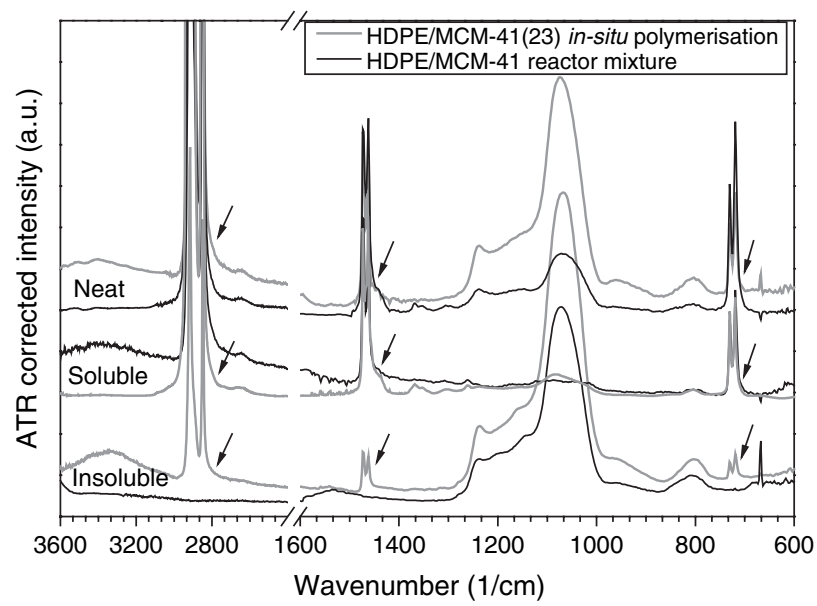

Fig. 5. FTIR spectra in ATR mode for HDPE/MCM-41(23) materials and corresponding soluble and insoluble fractions after extraction with boiling xylene for $10 \mathrm{~h}$. The arrows mark the strong absorption peaks due to the presence of polyethylene. outer PE chains from the inorganic material must be much more facilitated.

All these X-ray, TEM and FTIR observations are consistent with the fact that the in-situ polymerised materials under study are nanocomposites since the ethylene polymerisation has occurred at intrapores and, in addition, they exhibit a particle distribution at the nanometric scale. Therefore, these results corroborate several studies published in the literature that demonstrate the ability of M41S-type materials to immobilise zirconocene, or even bulkier metallocenes, and to allow the in-situ polymerisation of ethylene and propylene inside the pores and channels. ${ }^{17,27,37,38}$

\subsection{Thermal Analysis}

All the different specimens analysed are semicrystalline and develop the common orthorhombic lattice found in polyethylene crystallised under usual conditions. ${ }^{39}$ Accordingly, a melting process is observed in their calorimetric curves. Its location is practically constant independently of MCM-41 content in the nanocomposite, as seen in Table III. This similarity in the melting temperature values has been also described when different contents of $\mathrm{SiO}_{2}$ nanoparticles are incorporated to some polyethylenic matrices. ${ }^{40}$ Moreover, crystallinity values only change very slightly in this composition interval.

\subsection{Microhardness Measurements}

Figure 6 depicts the results found for the different nanocomposites exhibiting a significant microhardness increase as concentration of MCM-41 rises. The rigidity changes considerably with the incorporation of the mesoporous solid, leading to a considerable strengthening compared with neat HDPE. The almost invariable values found in all of the specimens for the $T_{\mathrm{m}}$ and crystallinity seem to indicate that the stiffness increase is attributed to the reinforcement effect of nanofiller and not to any variation in the amount or size of crystallites. Looking at values reported in the literature, it has to be said that the rigidity enhancement and, accordingly, the MH value found in the HDPE/MCM-41(28) nanocomposite (86 MPa) is much

Table III. Melting temperatures $\left(T_{\mathrm{m}}\right)$ and crystallinity values $\left(f_{\mathrm{c}}\right)$ estimated from enthalpies of first melting obtained through DSC experiments. The enthalpy has been normalised for the actual amount of polymer existing in each specimen.

\begin{tabular}{lcc}
\hline Nanocomposite & $T_{\mathrm{m}}\left({ }^{\circ} \mathrm{C}\right)$ & $f_{\mathrm{c}}$ \\
\hline HDPE & 132 & 0.53 \\
HDPE/MCM-41(3) & 131 & 0.52 \\
HDPE/MCM-41(4) & 133 & 0.53 \\
HDPE/MCM-41(6) & 132 & 0.53 \\
HDPE/MCM-41(14) & 132 & 0.60 \\
HDPE/MCM-41(23) & 132 & 0.55 \\
HDPE/MCM-41(28) & 132 & 0.51 \\
\hline
\end{tabular}


higher than that presented by a polyethylene-derivative with $60 \mathrm{wt} \%$ in glass fibre ${ }^{9}(21 \mathrm{MPa})$. This observation implies that these novel self-reinforced nanocomposites can achieve higher rigidity with filler loads lower than those incorporated in conventional microcomposites, leading, accordingly, to cheaper and lighter materials. The values reported in polyethylene-derivatives reinforced with $\mathrm{SiO}_{2}$ nanoparticles point out that the Young modulus decreases for $\mathrm{SiO}_{2}$ contents higher than $8 \mathrm{wt} \%$, due to formation of aggregates. ${ }^{40}$ However, the highest rigidity values here obtained are observed at the highest nanofiller contents because of the absence of large-size aggregates. The results here reported are also in agreement with those found in some other polymeric matrices where MCM-48 has been used instead of MCM-41 derivatives. ${ }^{28,29}$

Figure 6 also points out an analogous dependence on MCM-41 composition between MH values and those estimated from DMTA for $\mathrm{E}^{\prime}$ at the same temperature, indicating a perfect correlation between superficial and bulk responses for these nanocomposites. $\mathrm{MH}$ evaluates primarily the resistance of the polymer to plastic deformation, providing an idea about local strain. However, several effects can be distinguished in these measurements: ${ }^{33}$ an elastic deformation, a permanent plastic one and a viscoelastic time-dependent contribution. Therefore, though microhardness measurements are performed at the surface of films and, consequently, are related to the superficial mechanical response, they are also related to other bulk properties, as seen in these nanocomposites for the elastic modulus. Moreover, its determination has important advantages: its quickness, the small amount of material required, and its use as a rapid and reliable method of determining inhomogeneities caused during the processing of polymers and composites materials. ${ }^{9,12,41}$ Accordingly, several MH measurements were performed at different sections within the films to check the MCM-41 distribution homogeneity. All of the nanocomposites are rather homogeneous,

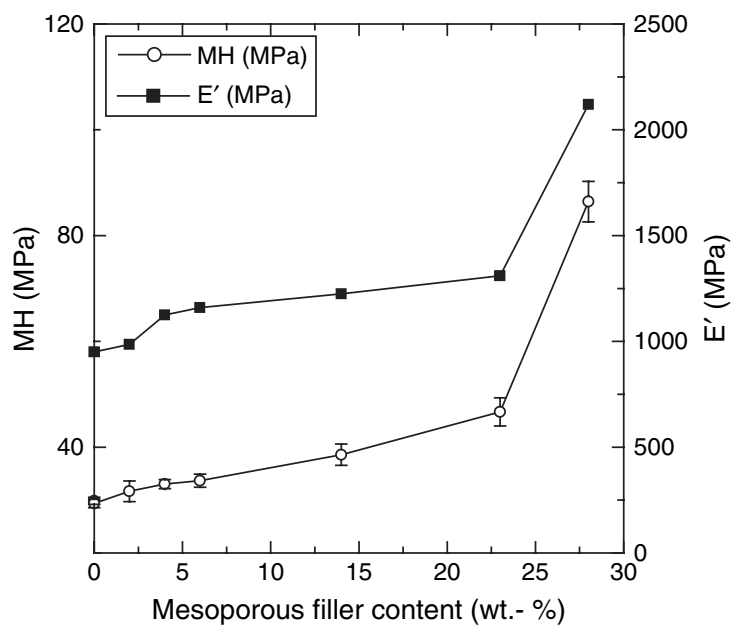

Fig. 6. Dependence of microhardness (MH, left $Y$ axis) and storage modulus ( $\mathrm{E}^{\prime}$, right $Y$ axis) on MCM-41 contents of the nanocomposites. although the material heterogeneity is slightly larger as nanofiller content increases and, consequently, error bars are enlarged (see Fig. 3). These results are in agreement with those previously discussed.

\subsection{Thermogravimetric Analysis}

Once novel self-reinforced materials have been prepared and the analysis of mechanical response has shown promising characteristics, the study of their thermal stability is a rather important aspect to be evaluated. Figure 7 shows the results obtained from thermogravimetry, indicating that the beginning of degradation is shifted to lower temperature as MCM-41 content increases in the nanocomposites. This shows the catalytic effect of MCM-41 in the degradation process of PE and illustrates the third role played by MCM-41 in the corresponding PE/MCM41( $x$ ) nanocomposites. A similar effect has been found by Marcilla ${ }^{42}$ et al. when studying the degradation of PE under $\mathrm{N}_{2}$, in the presence and absence of mesoporous MCM-41, by thermogravimetric analysis (TGA). Aguado ${ }^{43}$ et al. have also shown the efficiency of mesoporous aluminosilicate MCM-41 as promoter towards degradation of polyolefins into liquid fuels. Nowadays, this might be quite interesting in relation to the degradation of these polyolefins into basic petrochemicals as feedstock or fuel for downstream processes after their life service, making these self-reinforced polyolefinic materials environmentally welcome and with an added value. ${ }^{44}$ Furthermore, the amount of inorganic solids can also be estimated from these thermogravimetric curves. The contents agree rather well with those obtained from FTIR analysis, as listed in Table I.

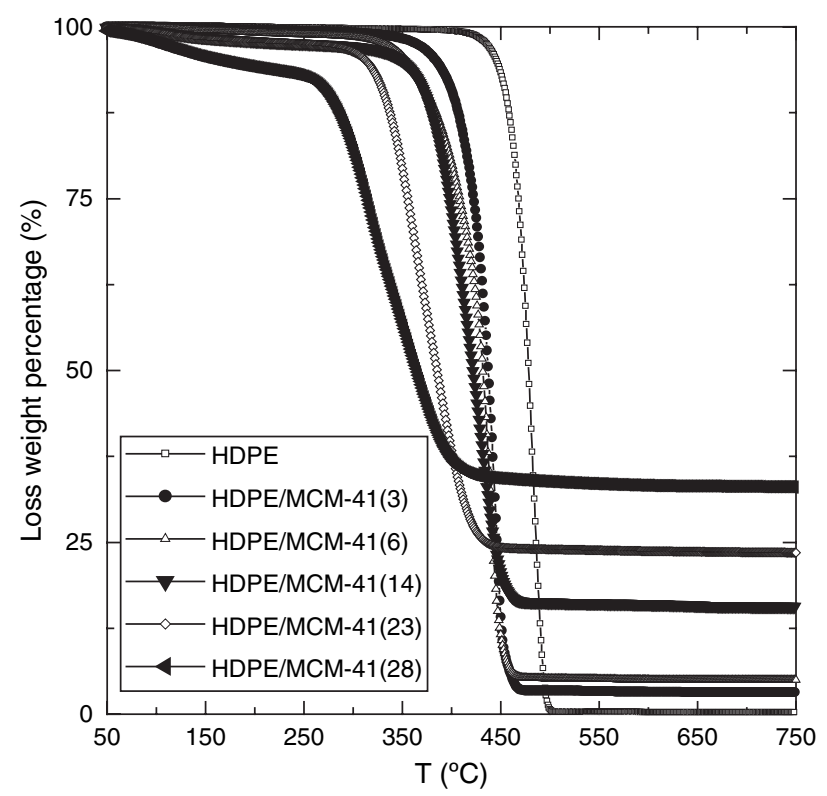

Fig. 7. Thermogravimetric profiles for the HDPE/MCM-41(x) nanocomposites. 


\section{CONCLUSIONS}

The use of mesoporous MCM-41 as catalyst carrier and its further presence in the final material is an effective route for the successful preparation of nanocomposites by in-situ supported polymerisation of ethylene within their pores and channels. These confined synthetic conditions lead to special interactions between the MCM-41 material and the resultant polyethylene, as shown by FTIR analysis. The careful selection of the experimental conditions allows the production of semicrystalline hybrid materials within a large range of nanofiller concentrations, whose crystallites at first approximation exhibit rather similar characteristics. These novel self-reinforced nanocomposites present an increase in rigidity, as well as an easier degradability because of the additional role of mesoporous MCM-41 as promoter for PE degradation. Therefore, their use may lead to cheaper, lighter and more environmentally friendly materials.

Studies aiming for a comprehensive evaluation of the crystalline and thermal characteristics and of the viscoelastic behaviour are currently in progress.

Acknowledgments: The authors are grateful for the financial support of the Exchange Collaboration Program CSIC/GRICES (projects 2005PT0033 and Proc. 4-1-1 Espanha 2006/2007, respectively), Fundação para a Ciência e a Tecnologia (FCT), (Projecto PDCT/CTM/66408/2006) and Ministerio de Educación y Ciencia (project MAT200500228). João M. Campos thanks the FCT for his Ph.D. scholarship (ref. SFRH/BD/16547/2004). The synchrotron work was supported by the European Community - Research Infrastructure Action under the FP6 "Structuring the European Research Area" Programme (through the Integrated Infrastructure Initiative "Integrating Activity on Synchrotron and Free Electron Laser Science"), contract RII3-CT-2004-506008 (IA-SFS). We thank the collaboration of the HASYLAB personnel in the soft-condensed matter beamline A2, especially Dr. S. S. Funari.

\section{References and Notes}

1. A. Okada and A. Usuki, Mater. Sci. Eng. C3, 109 (1995).

2. J. W. Gilman, Appl. Clay Sci. 15, 31 (1999).

3. J. W. Gilman, C. L. Jackson, A. B. Morgan, R. Harris, Jr., E. Manias, E. P. Giannelis, M. Wuthenow, D. Hilton, and S. H. Phillips, Chem. Mater. 12, 1866 (2000).

4. D. Porter, E. Metcalfe, and M. J. K. Thomas, Fire Mater. 24, 45 (2000).

5. M. Zanetti, S. Lomakin, and G. Camino, Macromol. Mater. Eng. 279, 1 (2000).

6. S. P. Armes, Polym. News 20, 233 (1995).

7. D. Y. Godovski, Adv. Polym. Sci. 119, 79 (1995).

8. D. M. Laura, H. Keskkula, and J. W. Barlow, Polymer 41, 7165 (2000).

9. M. L. Cerrada, R. Benavente, and E. Pérez, Macromol. Chem. Phys. 202, 2686 (2001).
10. M. L. Cerrada, R. Benavente, and E. Pérez, Macromol. Chem. Phys. 203, 718 (2002).

11. K. W. Cho, D. W. Kim, and S. Yoon, Macromolecules 36, 7652 (2003).

12. J. M. Martínez-Burgos, R. Benavente, E. Pérez, and M. L. Cerrada, J. Polym. Sci. Polym. Phys. 41, 1244 (2003).

13. G. Kickelbick, Prog. Polym. Sci. 28, 83 (2003).

14. W. Kaminsky, A. Funck, and K. Wiemann, Macromol. Symp. 239, 1 (2006).

15. K.-S. Lee, C.-G. Oh, J.-H. Yim, and S.-K. Ihm, J. Mol. Catal. A-Chem. 159, 301 (2000).

16. X. Zhang and L. C. Simon, Macromol. Mater. Eng. 290, 573 (2005).

17. H. Nakajima, K. Yamada, Y. Iseki, S. Hosoda, A. Hanai, Y. Oumi, T. Teranishi, and T. Sano, J. Polym. Sci., Part B: Polym. Phys. 41, 3324 (2003).

18. J. He, X. Duan, D. G. Evans, and R. F. Howe, J. Porous Materials 9, 49 (2002).

19. S. Ray, G. Galgali, A. Lele, and S. Sivaram, J. Polym. Sci., Part A: Polym. Chem. 43, 304 (2005).

20. Z. Ye, S. Zhu, W.-J. Wang, H. Alsyouri, and Y. S. Lin, J. Polym. Sci., Part B: Polym. Phys. 41, 2433 (2003).

21. K. Kageyama, J. Tamazawa, and T. Aida, Science 285, 2113 (1999).

22. K.-S. Lee, C.-G. Oh, J.-H. Yim, and S.-K. Ihm, J. Mol. Catal. A: Chem. 159, 301 (2000)

23. Y. S. Ko and S. I. Woo, Macromol. Chem. Phys. 202, 739 (2001).

24. Y. S. Ko and S. I. Woo, J. Polym. Sci. A: Polym. Chem. 41, 2171 (2003).

25. P. Kumkaew, S. E. Wanke, P. Praserthdam, C. Danumah, and S. Kaliaguine, J. Appl. Polym. Sci. 87, 1161 (2003).

26. P. Kumkaew, L. Wu, P. Praserthdam, and S. E. Wanke, Polymer 44, 4791 (2003).

27. X. Dong, L. Wang, G. Jiang, Z. Zhao, T. Sun, H. Yu, and W. Wang, J. Mol. Catal. A: Chem. 240, 239 (2005).

28. J. He, Y. Shen, J. Yang, D. G. Evans, and X. Duan, Chem. Mater. 15, 3894 (2007).

29. J. He, Y. Shen, and D. G. Evans, Microp. Mesop. Mater. 109, 73 (2008).

30. J. Lin and X. Wang, Polymer 48, 318 (2007).

31. J. M. Campos, J. P. Lourenço, M. R. Ribeiro, and A. Fernandes, J. Mol. Catal. A-Chem 277, 93 (2007)

32. B. Wunderlich, Macromolecular Physics, Academic Press, New York (1980), Vol. 3, p. 42.

33. F. J. Baltá-Calleja, Adv. Polym. Sci. 66, 117 (1985).

34. Ph. Dubois, M. Alexandre, and R. Jérôme, Macromol. Symp. 194, 13 (2003).

35. D. Lee, H. Kim, K. Yoon, K. E. Min, K. H. Seo, and S. K. Noh, Sci. Techn. Adv. Mater. 6, 457 (2005).

36. E. M. Flanigen, Zeolite Chemistry and Catalysis, ACS Monograph (1976), Vol. 171, p. 96.

37. T. Sano and Y. Oumi, Catalysis Surveys From Asia 8, 295 (2004).

38. X. Dong, L. Wang, W. Wang, H. Yu, J. Wang, T. Chen, and Z. Zhao, Eur. Pol. J. 41, 797 (2005).

39. D. C. McFaddin, K. E. Russel, G. Wy, and R. D. Heyding, J. Polym. Sci., Polym. Phys. 31, 175 (1993).

40. E. Kontou and M. Niaounakis, Polymer 47, 1267 (2006).

41. P. A. Engel and M. D. Derwin, Microindentation Techniques in Materials Science and Engineering, American Society of Testing and Materials, Philadelphia, PA (1984).

42. A. Marcilla, A. Gómez-Siurana, S. Menargues, R. Ruiz-Femenia, and J. C. García-Quesada, J. Anal. Appl. Pyrolysis 76, 138 (2006).

43. J. Aguado, D. P. Serrano, M. D. Romero, and J. M. Escola, Chem. Commun. 6, 725 (1996).

44. S. Chaianansutcharit, R. Katsutath, A. Chaisuwan, T. Bhaskar, A. Nigo, A. Muto, and Y. Sakata, J. Anal. Appl. Pyrolysis 80, 360 (2007).

Received: XX Xxxx xxxx. Accepted: XX Xxxx xxxx. 